\title{
Efecto del coadyuvante siliconado e insecticidas en el control del chanchito blanco de la vid, Pseudococcus viburni (Hemiptera: Pseudococcidae)
}

\author{
Luis Sazo, Jaime E. Araya ${ }^{1}$ y José de la Cerda \\ Facultad de Ciencias Agronómicas, Universidad de Chile, Casilla 1004, Santiago, Chile
}

\begin{abstract}
L. Sazo, J.E. Araya, and J. de la Cerda. 2008. Effect of a siliconate coadjuvant and insecticides in the control of mealybug of grapevines, Pseudococcus viburni (Hemiptera: Pseudococcidae). Cien. Inv. Agr. 35(2):215-222. The effect of a siliconate coadjuvant + poliether (Break) at 50,75, and $100 \mathrm{~mL} \cdot \mathrm{hL}^{-1}$, along with standard insecticides, was studied during the 2005-2006 growing season on Pseudococcus viburni mealybug control in two commercial vineyards in the central valley of the Metropolitan Region of Chile. These vineyards were naturally infested with mealybugs the previous season. A control program included three insecticide sprays, chlorpyrifos at post-harvest (autumn), and before cluster filling (summer), and diazinon when shoots were $10-15 \mathrm{~cm}$ long in the spring. A standard diazinon treatment without surfactant and a control treatment without spray were included. Levels of uninfested and infested clusters were determined at harvest on 100 clusters per experiment unit. Results were transformed by arcosen $\sqrt{ } \%$ and subjected to analysis of variance. Means were separated according to Tukey's test. On the basis of the results obtained, the application of trisiloxane in combination with polyether (Break) did not improve the effectiveness of chemical control in areas with a moderate to high infestation of $P$. viburni.
\end{abstract}

Key words: Adjuvant, Break, chlorpyrifos, diazinon, grape mealybug, surfactant, Vitis vinifera.

\section{Introducción}

El sector vitivinícola es un pilar importante en el comercio silvoagropecuario chileno hacia el mercado externo, con 112.056 ha destinadas a la producción de vino, y retornos de más de US\$ 258 millones por exportación en enero-abril de 2006 (ODEPA, 2007).

La vid (Vitis vinifera L.) tiene relativamente pocas plagas en Chile, especialmente las vides destinadas a la vinificación, en las que el daño cosmético tiene escasa importancia. Sin embargo, la mielecilla producida por chanchitos blancos (Pseudococcus spp; Hemiptera: Pseudococcidae) permite el desarrollo de fumagina (Cladosporium spp.),

Recibido 03 diciembre. Aceptado 12 mayo 2008.

1Dirigir correspondencia a Jaime Araya: jearaya@uchile.cl que confiere a los vinos características organolépticas indeseables (Sazo, 1995; Zaviezo, 2002). Además, estos hemípteros son vectores de algunos virus de la vid (ej. Grapevine corky bark virus, Grapevine virus B, and Grapevine leaf roll virus II) que disminuyen severamente la producción y el contenido de azúcar en los mostos (Auger y Esterio, 1998).

El chanchito blanco de la vid, Pseudococcus viburni (Signoret) $[=P$. obscurus Essig $=P$. affinis (Maskell)] es el pseudocóccido más abundante en los viñedos chilenos (Zaviezo, 2002). Se encuentra en muchas especies de plantas, incluyendo frutales, cactáceas suculentas, plantas forestales y ornamentales, malezas de hoja ancha, vegetación natural arbustiva, hortalizas y forrajeras, desde la III a la IX Región administrativa de Chile (Prado, 2001). A pesar de ser una especie cosmopolita, tiene gran importancia cuarentenaria, y puede 
ser la causa de rechazos y cierre de mercados externos.

Las hembras tienen tres estadíos ninfales y los machos sólo dos, con estados adicionales de prepupa y pupa. Los machos son alados, pequeños, grises, con alas transparentes cubiertas por cera y tienen corta vida. Se distinguen de otros pseudocóccidos por un estilo abdominal más largo y redondeado en el extremo (Ciampolini et al., 2003).

Esta plaga inverna como huevo bajo el ritidomo de la vid. En primavera se pueden encontrar individuos bajo el ritidomo (ninfas de la primera generación y hembras adultas pre-ovipostura) o en la base de los brotes, cerca de los restos de las yemas (Sazo, 1995). Su hábito críptico hace difícil su control. La presencia de P. viburni en racimos infestados sólo se detecta visual y fácilmente a la cosecha, cuando es muy tarde para su control (Sazo, 1989; Geiger et al., 2001).

Tanto el momento de aplicación como los insecticidas utilizados para su control dependen de la etapa fenológica de la vid y del ciclo de vida de $P$. viburni. Según el nivel de infestación, se hace control invernal, y posteriormente sobre brotes de $10 \mathrm{a} 15 \mathrm{~cm}$ de largo en primavera, antes del cierre de racimo en verano y en postcosecha (Daane et al., 2004). El principal objetivo es controlar los estados ninfales expuestos. Sin embargo, existen criterios variables sobre la metodología y momento óptimos para eliminar a los estados ninfales expuestos. Además, es necesario considerar las tolerancias máximas de residuos de los plaguicidas como asimismo la época de cosecha de los cultivares.

El tratamiento de postcosecha, a menudo clorpirifos más aceite mineral al $0,5 \%$, se dirige a controlar los estados móviles (ninfas y hembras adultas) en el follaje y racimos no cosechados y en al tronco antes que depositen todos los huevos. Como alternativa, se pueden aplicar metidathion, profenofos, diazinon, o buprofezin más aceite mineral o surfactante siliconado (Sazo, 1995).

El tratamiento de primavera se dirige a los estados móviles de la primera generación, que ocurre principalmente en la base de los brotes. Sin embargo, este tratamiento tiene efectividad variable, debido al largo período de eclosión de los huevos invernantes y a la prolongada (4 a 5 semanas) emergencia de las ninfas. Además, la mayoría de las ninfas se encuentran protegidas bajo la corteza. Se utilizan insecticidas como diazinon, carbaryl o imidacloprid. El uso de clorpirifos está limitado por fitotoxicidad en esta etapa fenológica de la vid (Sazo, 1995). Es importante considerar que diazinon no se puede utilizar desde diciembre de 2007 en los estados miembros de la Comunidad Económica Europea (DOCEE, 2007).

Aproximadamente un $30 \%$ del total de la población de chanchitos blancos se encuentra al interior de los racimos, y la aplicación antes del cierre de racimos es la última oportunidad para alcanzar estos individuos (Geiger et al., 2001). En esta época del desarrollo de la vid se hacen aplicaciones convencionales de imidacloprid o clorpirifos.

Algunos coadyuvantes compatibles con insecticidas permiten mejorar la cobertura y eficacia de la aplicación. Así es posible disminuir el uso de insecticidas, y minimizar las pérdidas por evaporación y deriva. Al mismo tiempo se favorece una mejor penetración del ingrediente activo a través de la cutícula del insecto y las estructuras de la planta (Rinehold y Jenkins, 2006). Estos productos podrían facilitar el control de $P$. viburni en vid, al mejorar el cubrimiento y penetración de la aspersión, lo que permitiría mejorar la eficiencia de los insecticidas, y reducir considerablemente los costos de aplicación. Este estudio tuvo por objetivo estudiar el efecto del coadyuvante siliconado trisiloxano en combinación con poliéter (Break), en el control químico de $P$. viburni en vid para vinificar.

\section{Materiales y métodos}

\section{Localidad}

Este estudio se realizó entre abril de 2005 y mayo de 2006 en Viña Concha y Toro, ubicada en Santa Isabel, Pirque ( $33^{\circ} 42^{\prime}$ lat. S, $70^{\circ} 35^{\prime}$ long. O), sobre un viñedo Pinot Noir de 9 años, plantado a 2,5 x 1,0 m, con 4.000 plantas $\mathrm{ha}^{-1}$, 
y en Viña Santa Rita, Alto Jahuel, Buin (334ㄴ lat. S, 70 44' long. O), en un viñedo Cabernet Sauvignon de 9 años, plantado a 1,7 x 1,0 m, con 5.882 plantas ha $^{-1}$. Ambos viñedos se ubican en el valle central en la Región Metropolitana. En la postcosecha de 2004-2005 se colectaron 50 hojas por unidad experimental para contar los estados móviles de $P$. viburni en cada muestra y conformar los bloques experimentales en función de los niveles de infestación existentes.

\section{Tratamientos}

Elefectodel coadyuvante siliconado, trisiloxano $\left(750 \mathrm{~g} \cdot \mathrm{L}^{-1}\right)+$ poliéter $\left(250 \mathrm{~g} \cdot \mathrm{L}^{-1}\right)($ Break SL, BASF, Santiago, Chile) se estudió en dosis de 50,75 y $100 \mathrm{~mL} \cdot \mathrm{hL}^{-1}$, de producto formulado, en vides tratadas con clorpirifos $\left(120 \mathrm{~mL} \cdot \mathrm{hL}^{-1}\right)$ y diazinon $\left(120 \mathrm{~g} \cdot \mathrm{hL} \mathrm{L}^{-1}\right)$, aplicados en los estados fenológicos de la vid que se indican en el Cuadro 1. Todas las aplicaciones se realizaron con una motobomba (Lévera, Santiago, Chile), con bomba de membrana Comet de $40 \mathrm{~L} \cdot \mathrm{min}^{-1}$, provista de pitón y una presión aproximada de 250 psi.

\section{Evaluación}

El nivel de infestación de $P$. viburni se evaluó en 100 racimos por unidad experimental a la cosecha (14 de marzo y 25 de abril de 2006 en Pirque y Buin, respectivamente), utilizando una escala de 0 a 3 donde 0 (sano) $=0$ presencia de individuos u ovisacos por racimo; 1 (leve) $=1$ a $5 ; 2$ (medio) $=6$ a 15 y 3 (alto) = más de 15 individuos $\mathrm{u}$ ovisacos por racimo, respectivamente. El grado de infestación (GI) se determinó mediante la ecuación de Townsend y Heuberguer (Wenda-Piesik et al., 1998),

$$
\mathrm{GI}=(\Sigma(\mathrm{n} * \mathrm{v})) * 100 / \mathrm{X} * \mathrm{~N}
$$

donde $\mathrm{n}=$ no. de racimos de cada categoría de evaluación, $\mathrm{v}=$ valor numérico de categoría, $\mathrm{X}=$ valor máximo de categorías, $\mathrm{N}=$ no. total de racimos.

\section{Diseño y análisis estadístico}

Los tratamientos se distribuyeron según un diseño de bloques completos al azar, con cinco

Cuadro 1. Epocas de aplicación, insecticidas y concentraciones utilizadas en los tratamientos contra el chanchito blanco (Pseudococcus viburni) de la vid.

Table 1. Timing, rates, and insecticides applied to control grapevine mealybug (Pseudococcus viburni).

\begin{tabular}{|c|c|c|c|}
\hline \multirow[t]{2}{*}{ Tratamientos } & \multicolumn{3}{|c|}{ Épocas de aplicación } \\
\hline & Postcosecha & $\begin{array}{c}\text { Primavera } \\
\text { (brotes de } 10 \text { a } 15 \mathrm{~cm} \text { ) }\end{array}$ & $\begin{array}{c}\text { Verano } \\
\text { (pre-cierre de racimos) }\end{array}$ \\
\hline Testigo & Agua & Agua & Agua \\
\hline Estándar & $\begin{array}{l}\text { Clorpirifos } 120 \mathrm{~mL} \\
+ \text { aceite mineral } 0,5 \%\end{array}$ & Diazinon $120 \mathrm{~g}$ & Clorpirifos $120 \mathrm{~mL}$ \\
\hline Break $50 \mathrm{~mL}$ & $\begin{array}{l}\text { Clorpirifos } 120 \mathrm{~mL} \\
+ \text { Break } 50 \mathrm{~mL}\end{array}$ & $\begin{array}{l}\text { Diazinon } 120 \mathrm{~g} \\
+ \text { Break } 50 \mathrm{~mL}\end{array}$ & $\begin{array}{l}\text { Clorpirifos } 120 \mathrm{~mL} \\
+ \text { Break } 50 \mathrm{~mL}\end{array}$ \\
\hline Break $75 \mathrm{~mL}$ & $\begin{array}{l}\text { Clorpirifos } 120 \mathrm{~mL} \\
+ \text { Break } 75 \mathrm{~mL}\end{array}$ & $\begin{array}{l}\text { Diazinon } 120 \mathrm{~g} \\
+ \text { Break } 75 \mathrm{~mL}\end{array}$ & $\begin{array}{l}\text { Clorpirifos } 120 \mathrm{~mL} \\
+ \text { Break } 75 \mathrm{~mL}\end{array}$ \\
\hline Break $100 \mathrm{~mL}$ & $\begin{array}{l}\text { Clorpirifos } 120 \mathrm{~mL} \\
+ \text { Break } 100 \mathrm{~mL}\end{array}$ & $\begin{array}{l}\text { Diazinon } 120 \mathrm{~g} \\
+ \text { Break } 100 \mathrm{~mL}\end{array}$ & $\begin{array}{l}\text { Clorpirifos } 120 \mathrm{~mL} \\
+ \text { Break } 100 \mathrm{~mL}\end{array}$ \\
\hline \multicolumn{4}{|c|}{$\begin{array}{l}\text { Fechas de aplicación: } \\
\text { Santa Isabel, }\end{array}$} \\
\hline $\begin{array}{l}\text { Pirque } \\
\text { Alto Jahuel, }\end{array}$ & 22 abril & 17 octubre & 19 diciembre \\
\hline Buin & 09 mayo & 21 octubre & 20 diciembre \\
\hline \multicolumn{4}{|l|}{ Cubrimiento: } \\
\hline Volumen, $L \cdot h a^{-1}$ & 2000 & 600 & 2000 \\
\hline
\end{tabular}


repeticiones. La unidad experimental tuvo 24 plantas distribuidas en cuatro hileras. Para evitar efectos de borde se muestreó en las ocho plantas de las dos hileras medias de cada unidad experimental. Los resultados se sometieron a análisis de varianza y los promedios se separaron de acuerdo con la prueba de comparación múltiple de Tukey ( $\leq 50,05$ ), utilizando el programa computacional GYE (C.E. Rauld, Universidad de Chile, Santiago, Chile). Previo a los análisis, los valores porcentuales se normalizaron por arcoseno $\sqrt{ } \%$.

\section{Resultados y discusión}

Hubo una diferencia considerable en el grado de infestación obtenido entre ambos viñedos, con una infestación mayor en Pinot Noir que en Cabernet Sauvignon. No obstante, en ambos viñedos se encontraron diferencias significativas $(\mathrm{p} \leq 0,05)$ de racimos sanos y nivel de infestación entre el testigo tratado con agua y los tratamientos con insecticidas (Cuadro 2).

Tanto el tratamiento estándar como aquellos con diversas concentraciones de coadyuvante presentaron un control similar de P. viburni. Sin embargo, ninguno de ellos controló totalmente esta plaga, corroborando la dificultad de obtener un control total, especialmente cuando las poblaciones son altas (Sazo, 1995). Por ejemplo, en Santa Isabel se obtuvo $60,6 \%$ de racimos sanos con el mejor tratamiento, resultado considerado insuficiente. Sin embargo, en Alto Jahuel, con un nivel moderado de infestación, se obtuvo $98 \%$ de racimos sanos.

Al agregar $50 \mathrm{~mL} \cdot \mathrm{hL}^{-1}$ de Break, el mojamiento y penetración del caldo fue adecuado y uniforme, mientras que con 75 y $100 \mathrm{~mL} \cdot \mathrm{hL}^{-1}$ de Break hubo escurrimiento superficial. La eficacia de la aplicación a estas dosis podría disminuir al dejar la planta con menos residuos insecticidas, y con ello, se obtendría un menor efecto de contacto y residual. Sin embargo, este escurrimiento en las aplicaciones con dosis altas de Break no se tradujo en un menor efecto insecticida sobre la plaga, y se mantuvo similar al del tratamiento estándar. Los resultados indican que tampoco se logró una mayor penetración del insecticida en la planta, lo que impidió un mayor control. Se debe aclarar que no necesariamente un coadyuvante contribuye a mejorar la acción insecticida de un producto específico. Por ejemplo, French et al. (1992), estudiando el efecto de tres surfactantes en el cubrimiento, persistencia y eficacia de clorpirifos contra Aphis gossypii en algodón, no obtuvieron resultados que demostraran un mejoramiento de las cualidades de los humectantes.

También se ha estudiado algún efecto insecticida de los coadyuvantes por si solos, con resultados variables. Por ejemplo, con 500 y $1000 \mathrm{~mL} \cdot \mathrm{hL}^{-1}$ del coadyuvante Silwet L-77 (i.a. copolímero de poliéter y silicona) sobre huevos y ninfas de $P$. maritimus en laboratorio, todas las ninfas murieron (Tipping et al., 2003). Según Barberá, 1989) a concentraciones $\geq 500 \mathrm{~mL} \cdot \mathrm{hL}^{-1}$. Los coadyuvantes tendrían cierto efecto insecticida/ acaricida, al asfixiar los individuos en forma similar al aceite mineral. En nuestro estudio, con una concentración máxima de $100 \mathrm{~mL} \cdot \mathrm{hL}^{-1}$ sólo hubo $6,7 \%$ de mortalidad, mientras que los huevos no fueron afectados a 100, $250 \mathrm{y}$ $500 \mathrm{~mL} \cdot \mathrm{hL}^{-1}$ (promedios no presentados). Esta mortalidad baja de ninfas y huevos se debería a la cubierta cerosa pulverulenta que tienen los Pseudococcus, y a la masa algodonosa que los protege e impediría la acción insecticida del surfactante, que se observa claramente en ensayos con otras plagas agrícolas. El mismo producto mata en laboratorio, a concentraciones similares a las usadas en el campo, las pupas de Ceratitis capitata y Bactrocera dorsalis (Purcell y Schroeder, 1996). Vargas et al. (2002) estudiaron también el efecto acaricida de adyuvantes a $100 \mathrm{~mL} \cdot \mathrm{hL}^{-1}$, solos y con residuos de Thuringiensin, sobre el ácaro Tetranychus urticae, y no encontraron efecto, debido a la ausencia de translocación en las hojas donde se ubicaron las arañitas, aunque tampoco hubo diferencias con el control al aplicarse surfactante directamente sobre los ácaros.

Resultados similares a los nuestros se han obtenido con otras plagas. En laboratorio, Sieburth et al. (1998) estudiaron sobre repollo el efecto de tres coadyuvantes más aceite mineral sobre la mosquita blanca Bemisia argentifolii. A $50 \mathrm{~mL} \cdot \mathrm{hL}^{-1}$ de cada coadyuvante más aceite mineral al $1 \%$ no hubo efecto sobre los huevos, 
Cuadro 2. Niveles de infestación de racimos de Vitis vinifera cvs. Pinot Noir y Cabernet Sauvignon con chanchito blanco (Pseudococcus viburni) sometidos a un programa estándar de control con diferentes concentraciones de trisiloxano + poliéter (Break).

Table 2. Mealybug (Pseudococcus viburni) infestation levels on Vitis vinifera clusters cvs. Pinot Noir and Cabernet Sauvignon, which were subjected to a standard control program using different concentrations of trisiloxano + polieter (Break).

\begin{tabular}{|c|c|c|c|c|}
\hline \multirow[t]{2}{*}{ Tratamientos } & \multicolumn{4}{|c|}{ Racimos (\%) según nivel de infestación } \\
\hline & Sano & Leve & Regular & Severo \\
\hline & \multicolumn{4}{|c|}{ Santa Isabel, cv. Pinot Noir } \\
\hline Testigo & $1,0 \mathrm{a}^{1}$ & $6,0 \mathrm{a}^{1}$ & $8,6 \mathrm{a}^{1}$ & $84,4 \mathrm{a}^{1}$ \\
\hline Estándar & $51,2 \mathrm{~b}$ & $22,8 \mathrm{~b}$ & $8,8 \mathrm{a}$ & $17,2 \mathrm{~b}$ \\
\hline Break $50 \mathrm{~mL}$ & $60,6 \mathrm{~b}$ & $17,2 \mathrm{~b}$ & $10,8 \mathrm{a}$ & $11,4 \mathrm{~b}$ \\
\hline Break 75 mL & $58,4 \mathrm{~b}$ & $15,4 \mathrm{~b}$ & $11,8 \mathrm{a}$ & $14,4 \mathrm{~b}$ \\
\hline \multirow[t]{2}{*}{ Break $100 \mathrm{~mL}$} & $52,0 \mathrm{~b}$ & $21,2 \mathrm{~b}$ & $15,0 \mathrm{a}$ & $11,8 \mathrm{~b}$ \\
\hline & \multicolumn{4}{|c|}{ Alto Jahuel, Cabernet Sauvignon } \\
\hline Testigo & $64,6 \mathrm{a}^{1}$ & $15,8 a^{1}$ & $7,0 \mathrm{a}^{1}$ & $12,6 \mathrm{a}^{1}$ \\
\hline Estándar & $92,2 \mathrm{~b}$ & $5,0 \mathrm{~b}$ & $1,8 \mathrm{ab}$ & $1,0 \mathrm{~b}$ \\
\hline Break $50 \mathrm{~mL}$ & $96,2 \mathrm{~b}$ & $2,8 \mathrm{~b}$ & $0,4 \mathrm{~b}$ & $0,6 \mathrm{~b}$ \\
\hline Break 75 mL & $96,0 \mathrm{~b}$ & $2,8 \mathrm{~b}$ & $0,6 \mathrm{~b}$ & $0,6 \mathrm{~b}$ \\
\hline Break $100 \mathrm{~mL}$ & $98,0 \mathrm{~b}$ & $1,6 \mathrm{~b}$ & $0,4 \mathrm{~b}$ & $0,0 \mathrm{~b}$ \\
\hline
\end{tabular}

${ }^{1}$ Promedios con letras iguales no son estadísticamente diferentes según la prueba de comparación múltiple de Tukey ( $\left.\mathrm{p}=0,05\right)$.

${ }^{1}$ Means followed by the same letters are not statistically different, according to Tukey's multiple range test $(p=0.05)$.

pero sí sobre ninfas de primer estadío. La emergencia de adultos desde las pupas tratadas fue baja con todos los coadyuvantes. Estos resultados indican que los coadyuvantes son ineficientes para matar huevos, lo que sumado a que los huevos de $P$. viburni estaban cubiertos por una masa algodonosa en nuestro estudio, y bajo la corteza en tratamientos de postcosecha y primavera, explican el efecto nulo de Break en esta plaga.

Shapiro et al. (1998) compararon la efectividad de la bacteria Bacillus thuringiensis con y sin coadyuvante contra la larva del minador de los cítricos Phyllocnistis citrella. El surfactante aumentó el ingreso de las soluciones en las galerías foliares, y con ello la actividad de $B$. thuringiensis hasta causar $90 \%$ de mortalidad. Sin embargo, en un ensayo de campo, la mortalidad bajó a 50\%, y no se redujo significativamente el nivel de daño en las hojas ni el número de larvas por hojas a los 14 días, en comparación con un tratamiento convencional sin surfactante. Howell y Reed (1999) no encontraron diferencias al usar insecticidas con o sin coadyuvantes contra Lygus lineolaris en algodón, con resultados similares a los de nuestro ensayo. Sin embargo, Cowles et al. (2000) encontraron un gran efecto acaricida de la solución con adyuvante $\left(500 \mathrm{~mL} \cdot \mathrm{hL}^{-1}\right)$ sobre T. urticae, en comparación con el tratamiento estándar y el testigo.

Los insecticidas utilizados en este estudio actúan por contacto, ingestión e inhalación, y la aspersión no llegan a sectores de la planta donde suelen haber muchos individuos de $P$. viburni. Los resultados también pueden ser afectados por las características de la planta de vid (ritidomo abundante, racimos apretados y junto a los brazos). Pinot Noir, a diferencia de Cabernet Sauvignon, tiene ritidomo grueso, racimos muy apretados y junto a la madera de los brazos. Según Campos y Sazo (1983), P. viburni causa daño principalmente en racimos apretados. Según los resultados indicados en el Cuadro 2, pareciera que las características morfológicas de Cabernet Sauvignon, con el envés de las hojas con numerosos tricomas, tampoco favorecen un control más eficiente con coadyuvantes. No obstante, los niveles de infestación alta y media obtenidos en los testigos en este trabajo son atribuibles principalmente al manejo de estos viñedos en temporadas precedentes a este estudio. Además, las oportunidades de cada aplicación en este ensayo pueden también haber influido en los resultados finales. En las aplicaciones en poscosecha, parte de la plaga 
se encontró bajo el ritidomo (especialmente en Pinot Noir), y ninguno de los tratamientos con Break debe haber penetrado suficientemente; en cambio, en las hojas de Cabernet Sauvignon la gran cantidad de tricomas hace difícil obtener un buen mojamiento. Durante la brotación, en ambas localidades permanecían aún bajo el ritidomo una cantidad variable de ninfas de $P$. viburni, tanto o más que las establecidas en los brotes. Por último, para la aplicación de verano, los racimos de Pinot Noir presentaron bayas más grandes que los de Cabernet Sauvignon, cultivar que desarrolla racimos comparativamente más apretados, lo que seguramente afectó en la acción de los insecticidas.

En general, si se usan coadyuvantes para aplicar cualquier producto se recomienda un alto volumen de agua para aumentar su penetración y cubrir mejor la planta. Pease y Zalom (2006) encontraron efectos distintos de los surfactantes sobre Macrosiphum euphorbiae en tomate según el mojamiento, iguales a los tratamientos estándar en aplicaciones de 467 $\mathrm{L} \cdot \mathrm{ha}^{-1}$, pero estadísticamente mejores con el doble de mojamiento, al aumentar la superficie de cubrimiento. Al usar volúmenes mayores de aplicación, Curkovic et al. (2007) obtuvieron también un mayor control de $P$. longispinus con dos detergentes agrícolas. Al aplicar 1.500 L ha ${ }^{-1}$ en el campo, el uso de coadyuvante junto con la mitad de la dosis utilizada para controlar a P. longispinus en caqui, Diospyros kaki. Gaskin et al. (1996) controlaron igual que con la dosis máxima del insecticida sin coadyuvante, y mejor que con la mitad del insecticida aplicado solo. Para uva vinífera, los volúmenes de aplicación pueden reducirse si se usan los adyuvantes apropiados, sin comprometer la eficacia del insecticida (Gaskin et al., 2002).

Según Holloway et al. (2000), el coadyuvante siliconado mejora la cobertura de la aspersión sobre las hojas, y Western et al. (1999) indican que los coadyuvantes reducen la proporción de microgotas, y que hay una relación inversa entre la cantidad de coadyuvante y el desarrollo de deriva.

En este estudio se utilizaron volúmenes de agua similares a los usados por los vitivinicultores nacionales en distintas etapas fenológicas de la vid para una cobertura total. En cada aplicación, las hojas, brotes y zona del cuello quedaron totalmente cubiertos con el caldo de aspersión. En viñedos de California se recomiendan gastos de 1400 a $1900 \mathrm{~L}^{-1} \mathrm{~h}^{-1}$, niveles menores a los de este estudio, y Bentley et al. (2003) destacan que un buen cubrimiento es esencial para controlar pseudocóccidos, por lo que en nuestro trabajo se descarta el efecto de una mala distribución de los insecticidas aplicados en los resultados finales, y el mojamiento no habría incidido en el nulo efecto de Break obtenido.

Como conclusión, bajo las condiciones experimentales de este trabajo, con una infestación media a alta de $P$. viburni, la aplicación de trisiloxano en combinación con poliéter (Break) no mejoró la eficacia de un programa estándar de control químico, expresada en proporción de racimos sanos.

\section{Resumen}

Se estudió durante la temporada 20052006 el efecto del coadyuvante siliconado trisiloxano en combinación con poliéter (Break) en concentraciones de 50, 75 y 100 $\mathrm{mL} \cdot \mathrm{hL}^{-1}$ junto con insecticidas en el control de chanchito blanco de la vid (Pseudococcus viburni) en dos viñedos comerciales de la Región Metropolitana de Chile, infestados naturalmente la temporada precedente, como parte de un programa normal de control, con clorpirifos en post-cosecha y previo al cierre del racimo (verano), y diazinon en brote de 10$15 \mathrm{~cm}$ en primavera. Se incluyó un tratamiento estándar de diazinon sin surfactante y un testigo sin aplicación. En cosecha, se determinaron los racimos sanos y con diversos grados de infestación, sobre 100 racimos por unidad experimental. Los resultados se transformaron por arcoseno $\sqrt{ }$ porcentaje y sometieron a análisis de varianza y prueba Tukey para separar promedios. Bajo las condiciones del ensayo, las concentraciones de Break no afectaron la eficacia de los tratamientos, expresada en porcentaje de racimos sanos.

Palabras clave: Break, chanchito blanco de la vid, chlorpirifos, coadyuvante, diazinon surfactante, Vitis vinifera. 


\section{Literatura citada}

Auger, J. y M. Esterio. 1998. Las enfermedades a virus de la vid: sintomatología, principales formas de transmisión y efectos en la producción y calidad de la uva y sus productos. Aconex 59:5-13.

Barberá, C. 1989. Pesticidas agrícolas, $4^{\mathrm{a}}$ ed, Editorial Omega, Barcelona, España, 603 p.

Bentley, W.J., L.G. Varela, F.G. Zalom, R.J. Smith, A.H. Purcell, P.A. Phillips, D.R. Haviland, K.M. Daane y M.C. Battany. 2003. UC IPM Pest Management Guidelines: Grape Mealybugs (Pseudococcus), www.ipm.ucdavis.edu/PMG/ r3023011811.html. (Consultado: mayo de 2008).

Campos, L. y L. Sazo. 1983. Plagas de la vid en Chile y su control, Universidad de Chile, Facultad de Ciencias Agrarias, Veterinarias y Forestales, Santiago, Chile, $151 \mathrm{p}$.

Ciampolini, D., D. Lunghini y G. Mocetti. 2003. Insidioso nemico della frutticoltura: Pseudococcus viburni. L'Informatore Agrario 1:57-60.

Cowles, R.S., E.A. Cowles, A.M. McDermott y D. Ramoutar. 2000. Inert formulation ingredients with activity: Toxicity of trisiloxane surfactant solutions to two spotted spider mites (Acari: Tetranychidae). J. Econ. Entomol. 93:180-188.

Curkovic, T., G. Burett y J.E. Araya. 2007. Evaluation of the insecticide action of two agricultural detergents against the long-tailed mealybug, Pseudococcus longispinus (Hemiptera: Pseudococcidae), in the laboratory. Agricultura Técnica (Chile) 67:422-430.

Daane, K., E. Weber y W. Bentley. 2004: Vine mealybug, formidable pest spreading through California vineyards, http://ucce.ucdavis.edu/ files/filelibrary/1650/14714.pdf. (Consultado: mayo de 2008).

DOCEE. 2007. Decisión de la Comisión (relativa a la autorización de uso de diazinon), Diario Oficial de la Comunidad Económica Europea (DOCEE) http://eur-lex.europa.eu/LexUriServ/site/es/oj/ 2007/1_148/1_14820070609es00090010.pdf. (Consultado: mayo de 2008).

French, N.M., S.B. Ramaswamy, D.B. Smith y D. Paroonagian. 1992. Effect of three adjuvants on coverage, persistence, and efficacy of ground-applied chlorpyrifos for suppression of cotton aphid (Homoptera: Aphididae). J. Econ. Entomol. 85:1347-1355.

Gaskin, R.E., D.W. Manktelow y G.S. Elliott. 2002. New adjuvant technology for pesticide use on wine grapes. New Zealand Plant Protection 55:154-158.
Gaskin, R.E., B.H. Rohitha y P.T. Holland. 1996. Control of insect pests in persimmon with spray oils, In: Proc. New Zealand Plant Protection Conf., New Zealand Plant Protection Society Inc., pp. 27-31, http://ww.nzpps.org/journal/49/ nzpp49_027.pdf. (Consultado: mayo de 2008).

Geiger, C., K. Daane, W.J. Bentley, G. Yokota y L. Martin. 2001: Sampling program for grape mealybugs improves pest management. California Agric. 55:19-28.

Holloway, P.J., M.C. Butler Ellis, D.A. Webb, N.M. Western, C.R. Tuck, A.L. Hayes y P.C.H., Miller. 2000. Effects of some agricultural tankmix adjuvants on the deposition efficiency of aqueous sprays on foliage. Crop Protection 19: 27-37.

Howell, M.S. y J.T. Reed. 1999. Effects of five different adjuvants added to five insecticides for control of tarnished plant bugs (Lygus lineonaris) in Mississippi cotton. Pages 10521054. In: Proc. 1999 Beltwide Cotton Conf., January 1999, Orlando, Florida, USA.

ODEPA. 2007. Vides: Superficie y producción. Oficina de Planificación Agrícola (ODEPA). Gobierno de Chile. www.odepa.cl. (Consultado: mayo de 2008).

Pease, C.G. y F.G. Zalom. 2006. Can adjuvants improve efficacy of organic insecticides? Control of Macrosiphum euphorbiae on fresh market tomatoes. Page 80. In: Abstracts $90^{\text {th }}$ Annual Meeting, Pacific Branch, Entomological Soc. of America. Maui, Hawaii.

Prado, E. 2001. Artrópodos y sus enemigos naturales asociados a plantas cultivadas en Chile. INIA, La Platina, Boletín Técnico No 169, Santiago, Chile, $207 \mathrm{p}$.

Purcell, M.F. y W.J. Schroeder. 1996. Effect of Silwett L-77 and diazinon on three tephritid fruit flies (Diptera: Tephritidae) and associated endoparasitoids. J. Econ. Entomol. 89:15661670.

Rinehold, J. y J. Jenkins. 2006. Spray-tank adjuvants. In: PNW Insect Management Handbook. http:// pnwpest.org/pnw/insects. (Consultado: mayo de 2008).

Sazo, L. 1989. Manejo de chanchitos blancos en parronales de uva de mesa. En: Manejo de Plagas y Enfermedades en Frutales y Uva de Mesa, Universidad de Chile, Facultad de Ciencias Agrarias y Forestales. Publicaciones Misceláneas Agrícolas 30:45-48.

Sazo, L. 1995. Control de chanchitos blancos en frutales de hoja caduca. En: Sanidad vegetal en frutales y vides, Universidad de Chile, Facultad de Ciencias Agrarias y Forestales. Publicaciones Misceláneas Agrícolas 41:60-63. 
Shapiro, J.P., W.J. Schroeder y P.A. Stansly. 1998. Bioassay and efficacy of Bacillus thuringiensis and an organosilicone surfactant against the citrus leafminer (Lepidoptera: Phyllocnistidae). Florida Entomol. 81: 201-210.

Sieburth, P.J., W.J. Schroeder y R.T. Mayer. 1998. Effects of oil and oil-surfactant combinations on silverleaf whitefly nymphs (Homoptera: Aleyrodidae) on collards. Florida Entomol. 81:446-450.

Tipping, C., V. Bikoba, G.J. Chandler y E.J. Mitcham. 2003. Efficacy of Silwett L-77 against several arthropod pests of table grape. J. Econ. Entomol. 96:246-250.

Vargas, R., B. Chapman y D. Penman. 2002. Factores que influyen en la respuesta de Tetranychus urticae Koch (Acarina: Tetranychidae) a thuringiensin. Agricultura Técnica (Chile) 62:3-14.
Wenda-Piesik, A. y D. Piesik. 1998. The spring cereal for preferences of Oulema spp. in pure and mixed crops. Elec. J. Pol. Agric. Univ., Agronomy 1:1. http://www.ejpau.media.pl/series/volume1/ agronomy/art-04.pdf. (Consultado: mayo de 2008).

Western, N.M., E.C. Hislop, P.J. Holloway y D. Coupland. 1999. Drift reduction and dropletsize in sprays containing adjuvant oil emulsions. Pesticide Science 55: 633-637.

Zaviezo, T. 2002. Manejo integrado del chanchito blanco en viñedos. Páginas 24-33. En: Tópicos de Actualización en Viticultura y Enología. Pontificia Universidad Católica de Chile, Departamento de Fruticultura y Enología y Centrodel Vino, CEVIUC. Santiago, Chile. 254p. 